\title{
Spontaneous trimerization in two-dimensional antiferromagnets
}

\author{
Zi Cai, Shu Chen, and Yupeng Wang \\ Beijing National Laboratory for Condensed Matter Physics, Institute of Physics, \\ Chinese Academy of Sciences, Beijing 100080, P. R. China
}

(Dated: Received November 25, 2018)

\begin{abstract}
In this paper, we propose a novel quantum paramagnetic state in two-dimensional antiferromagnets: the spontaneously trimer state, which is constructed by the trimers of spins. Each trimer is a singlet state formed by three neighboring spins with SU(3) symmetry. A frustrated spin-1 Heisenberg model in the kagome lattice is investigated. By analogy to the pseudo-potential approach in the fractional quantum Hall effect (FQHE), we find that the trimer state provides a perfect description for the exact ground state of this model. Other interesting properties, such as the local excitations as well as magnetization plateaus have also been investigated.

PACS numbers: 75.10.Jm, 71.27.+a, 75.10.-b
\end{abstract}

The nature of quantum paramagnetic phases of the two-dimensional (2D) antiferromagnetic systems attracted considerable attention in the past twenty years because of its potential association with the physics of the cuprate superconductors [1]. Because of absence of long-range order in the quantum paramagnetic phase, a central problem is the classification of the phases and critical points. According to their symmetries, we could classify these phases into two classes. The first kind is known as "spin liquid state", which restores the $\mathrm{SU}(2)$ symmetry of the Neel state and does not break any symmetry of the original Hamiltonian. One of the well-known state in this class is the resonating valence bond (RVB) state 2], or the "spin liquid" state [3].

On the other hand, Read and Sachdev have presented another possibility [4] based on the Schwinger boson analysis on the $\mathrm{SU}(\mathrm{N})$ quantum antiferromagnets. Their key point is that the condensation of the instantons with the Berry phase leads to the spontaneous breaking of the spatial symmetry (translational or rotational symmetry) of the original Hamiltonian. Similar with the solid, this state possesses a short range order and thus is nominated as valence-bond solids (VBS) state. As shown in Ref. [4], the structure of the VBS state depends on $2 \mathrm{~S}(\bmod 4)$, where $\mathrm{S}$ is the spin of the $\mathrm{SU}(\mathrm{N})$ model. For the usual spin $1 / 2$ systems, corresponding VBS state is known as "dimer state", where two nearest spins form a singlet or a dimer: $(\uparrow \downarrow-\downarrow \uparrow) / \sqrt{2}$ and the overall state is the product of all those dimers.

The dimer state is first proposed as the exact ground state for the Majumdar-Ghosh(MG) model [5], which is an antiferromagnetic spin chain with nearest and nextnearest neighbor interactions. For a particular ratio of those exchange interactions, the model has a two-foldly degenerate dimer ground state. Inspired by the exact solution of the MG model, Shastry and Sutherland proposed a two dimensional model [6] known as ShastrySutherland (SS) model, whose ground state is the exact dimer state in 2D. It has been used in understanding the physical properties of $\mathrm{SrCu}_{3}\left(\mathrm{BO}_{3}\right)_{2}$ [7], which is topologically equivalent to the SS model.

Most above researches focus on the spin- $1 / 2$ system, because of its potential correlation with the high-Tc superconductivity. However, the cold atom systems have provided an ideal playground to investigate the high-spin strongly correlated system experimentally. For example, several proposals have been provided to realize the spin-1 Heisenberg model in the optical lattice [8]. Even in one dimension, new possibility emerges and a typical example is known as the Affleck-Kennedy-Lieb-Tasaki (AKLT) state [9]. This state breaks no symmetry of the Hamiltonian and is believed to provide a good description for the ground state of the pure spin-1 Heisenberg chain [10, 11]. As to the $2 \mathrm{D}$ case, the situation is apparently more complex, the VBS state in the spin-1 quantum paramagnetic phase could be either the dimer state [12] that spontaneously breaks the translational symmetry, or a generalized AKLT state [1] that only breaks the rotational symmetry.

In this paper, we present a novel VBS state named as "trimer state" for the spin-1 system in the 2D Kagome lattice. Such a state is a product of trimer singlets with each spin singlet formed by three spins with the total spin zero. Inspiring by the seminal work of Ref. [10], we constructed a frustrated Heisenberg model in a kagome lattice, and found that at a particular ratio of the different couplings, the "trimer state" provides a perfect approximation for the exact ground state of this model. Similar states have been discussed in an SU(3) spin tetrahedron chain [13-16] and in the kagome lattice with distorted coupling [17]. To our knowledge, we provide the first example in an isotropic $2 \mathrm{D} \mathrm{SU}(2)$ antiferromagnets with uniform coupling, where the spins are spontaneously trimerized. Then we investigated the excited properties and the magnetization plateaus in the applied magnetic field. It is believed that this state represents a novel class of the VBS state: $\mathrm{N}$ neighboring spins cluster together to form a singlet with $\mathrm{SU}(\mathrm{N})$ symmetry and spontaneously break the spacial symmetry. Within a cluster, each spin is maximally entangled with other spins. We call this kind of state as "cluster state" while the usual dimer state is the simplest case.

The ground state of the quantum antiferromagnetic Heisenberg model on the kagome lattice has been in- 


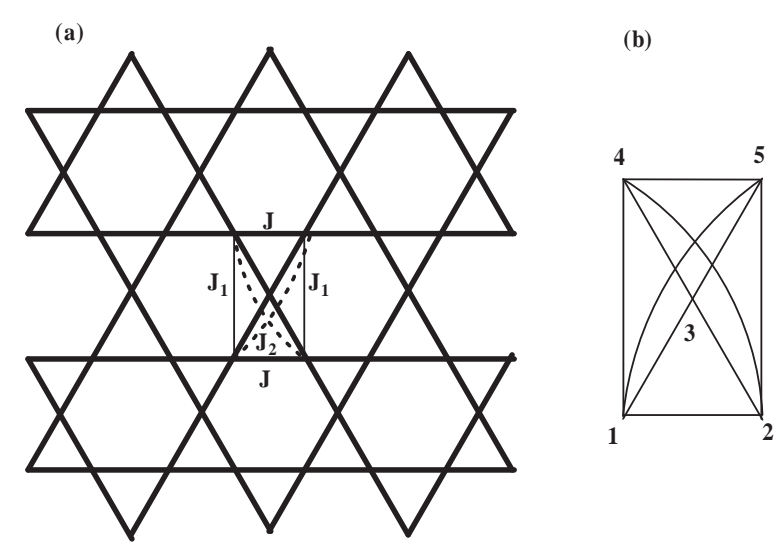

FIG. 1: (a) The structure of frustrated Heisenberg model in kagome lattice, with the nearest coupling $J$, the second nearest coupling $J_{1}$ and the third nearest coupling $J_{2}$. (b) One block consists of 5 sites and each one equally couples with others

tensively investigated by various methods, but is still far from being totally understood even for the spin- $1 / 2$ case. Recently, Laws et. al found a $\mathrm{Ni}^{2+}$-based material: $\mathrm{Ni}_{3} \mathrm{~V}_{2} \mathrm{O}_{8}$ [18], which is topologically equivalent to a frustrated spin-1 Heisenberg model in kagome lattice. The spin-1 model on the kagome lattice has been investigated in several works [19-22], but most of them focus on the uniaxial anisotropic case. Here we focus on the $\mathrm{SU}(2)$ case and introduce the second nearest $\langle\langle\mathbf{i} \mathbf{j}\rangle\rangle$ and the third nearest [ij] couplings $J_{1}$ and $J_{2}$. Explicitly, the Hamiltonian for this model is given by

$$
H=J \sum_{\langle\mathbf{i}, \mathbf{j}\rangle} \mathbf{S}_{\mathbf{i}} \cdot \mathbf{S}_{\mathbf{j}}+J_{1} \sum_{\langle\langle\mathbf{i}, \mathbf{j}\rangle\rangle} \mathbf{S}_{\mathbf{i}} \cdot \mathbf{S}_{\mathbf{j}}+J_{2} \sum_{[\mathbf{i}, \mathbf{j}]} \mathbf{S}_{\mathbf{i}} \cdot \mathbf{S}_{\mathbf{j}}
$$

with the structure schematically shown in Fig.1.

In this paper we would focus on the point $J=3 J_{1}=$ $3 J_{2}$ and investigate the ground state at this point by the "pseudopotential approach", which was first used to get the famous Laughlin wavefunction in the FQHE [23]. The analogy between the Heisenberg model and the FQHE was first introduced by Arovas et. al. [10] in the 1D case, where the Hamiltonian of a spin- 1 antiferromagnetic Heisenberg model is decomposed into the summation of projection operators

$$
H_{1}=\sum_{i} \mathbf{S}_{\mathbf{i}} \cdot \mathbf{S}_{\mathbf{i}+\mathbf{1}}=\sum_{i}\left[3 P^{2}(i, i+1)+P^{1}(i, i+1)-2\right] .
$$

Observing that the AKLT state [9] is the exact ground state of the first part of the summation of projection operators $\sum_{i} 3 P^{2}(i, i+1)$, they take it as a trial ground state of the spin-1 Heisenberg chain and consider the second part $\sum_{i} P^{1}(i, i+1)$ as a perturbation. It turns out that the AKLT state is a very good approximation of the exact ground state of Eq. (2) and numerical results show that the difference between the ground state energies of these two states is within $5 \%$. Recently, we generalized this method to the $2 \mathrm{D}$ spin- $1 J_{1}-J_{2}$ antiferromagnetic model[11], and found that at the maximal frustrated point $\left(J_{1}=2 J_{2}\right)$, the ground state could be described by a two-fold 2D generalized AKLT states, which completely agrees with the general prediction by the field theory 4 ], and was verified by the numerical results recently [24].

Next we use the similar method to investigate the ground state of Eq. (1). Notice that at the point $J=3 J_{1}=3 J_{2}$, Eq. (1) could be rewritten as the sum of identical blocks $\mathfrak{B}_{\alpha}$, as shown in Fig 1.(b). Each block is constructed by 5 spins and every spin is coupled to the other four spins identically, thus we have

$$
H / J_{1}=\sum_{\alpha} \mathfrak{B}_{\alpha} \quad \text { with } \quad \mathfrak{B}_{\alpha}=\sum_{\mathbf{i}, \mathbf{j} \in \alpha} \mathbf{S}_{\mathbf{i}} \cdot \mathbf{S}_{\mathbf{j}}
$$

where $\alpha$ denotes the $\alpha$ th block. Then we expand this block Hamiltonian by the projection operators of the total spin in a block. In terms of the projection operators, we have

$$
\mathfrak{B}_{\alpha}=\sum_{S=0}^{5} C_{S} \mathbf{P}_{\alpha}^{\mathbf{S}}-5 \quad \text { with } \quad C_{s}=S(S+1) / 2 .
$$

To make progress, we further decompose it as two part

$$
\mathfrak{B}_{\alpha}=\mathfrak{B}_{\alpha}^{0}+\mathfrak{B}_{\alpha}^{1},
$$

with $\mathfrak{B}_{\alpha}^{0}=15 \mathbf{P}_{\alpha}^{5}+10 \mathbf{P}_{\alpha}^{4}+6 \mathbf{P}_{\alpha}^{3}-5$ and $\mathfrak{B}_{\alpha}^{1}=3 \mathbf{P}_{\alpha}^{2}+\mathbf{P}_{\alpha}^{1}$. The operator $P_{\alpha}^{S}$ projects the spin state of the $\alpha$ th Block onto the subspace with total spin $S$. Dividing the original Hamiltonian into two parts is inspired by the success of Haldane's pseudopotential method in FQHE 23] and the model of the spin-1 chain [10]. Return to our spin model, now it is clear why we divided the Hamiltonian like this: notice that the coefficient decreases rapidly as $S$ descends, thus if we can find the exact ground state of $\mathfrak{B}_{0}=\sum_{\alpha} \mathfrak{B}_{\alpha}^{0}$, we can treat the left part $\mathfrak{B}_{1}=\sum_{\alpha} \mathfrak{B}_{\alpha}^{1}$ and investigate the properties of the ground state.

First we focus on the exact ground state of the model

$$
\mathfrak{B}_{0}=\sum_{\alpha}\left[15 \mathbf{P}_{\alpha}^{\mathbf{5}}+10 \mathbf{P}_{\alpha}^{4}+6 \mathbf{P}_{\alpha}^{3}-5\right]
$$

Since $\mathfrak{B}_{\alpha}^{0}$ is positive semidefinite, any state with the total spin of each block $S_{\alpha}^{T} \leq 2$ is the exact ground state of Eq.(5). It is not difficult to find that the only possibilities are the two-fold degenerate states as shown in Fig.2(a) and (b), where each block possesses a trimer singlet. Explicitly, the trimer singlet can be represented as

$$
|T\rangle=\frac{1}{\sqrt{6}} \sum_{\alpha, \beta, \gamma} \epsilon_{\alpha \beta \gamma}\left|\alpha_{i}, \beta_{j}, \gamma_{k}\right\rangle
$$

which is a singlet composed of three spins on neighboring sites $i, j$, and $k$ with total spin zero (Fig.2(c). Here $\alpha_{i}$ denotes the spin on site $i$ with the value $\alpha$ taking 1, 0 , or -1 and $\epsilon_{\alpha \beta \gamma}$ is an antisymmetric tensor. Because there 

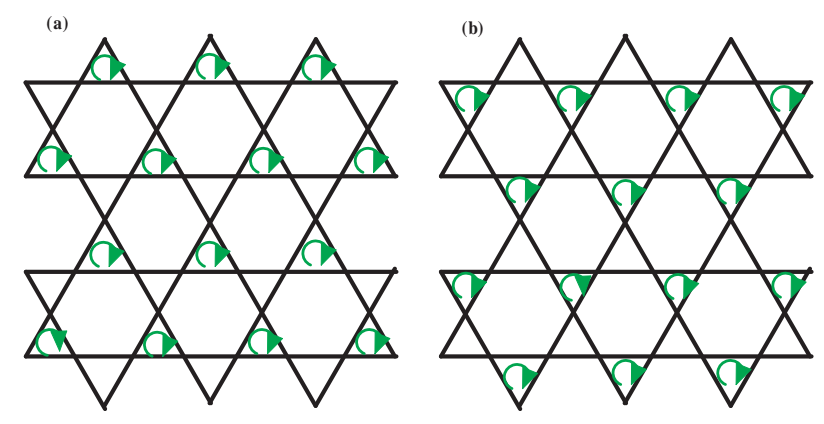

(1)

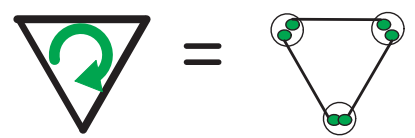

FIG. 2: (color online)(a) and (b): The two-fold degenerate ground states: $\left|\Psi_{a}\right\rangle$ and $\left|\Psi_{b}\right\rangle$. (c) The structure of one trimer: a AKLT chain with Length $\mathrm{L}=3$ and periodical boundary condition

exists a trimer singlet in each block, the total spin of each spin block could not be larger than 2. Therefore it is straightforward that we have found the exact ground states of $\mathfrak{B}_{0}$, or in other words, the trial ground state of our original Hamiltonian Eq. (3).

Now we will discuss the effect of the perturbation part. Since the trial ground states are two-fold degenerate, there seems to be a possibility that the perturbation would resonate these two trial ground states $\left|\Psi_{a}\right\rangle$ and $\left|\Psi_{b}\right\rangle$ to form a linear superposition and further lower the energy of the ground state. However, we would show this is not the case, at least in the thermodynamic limit. Let's calculate the nondiagonal term $\left\langle\Psi_{a}|H| \Psi_{b}\right\rangle$, it is not difficult to find that this nondiagonal term vanishes in the thermodynamic $\operatorname{limit}(N \rightarrow \infty)$, just like the $1 \mathrm{D} \mathrm{MG}$ model. So the perturbation would not shift the two-fold degeneracy of the trial ground states and we can safely conclude that $\left|\Psi_{a}\right\rangle$ and $\left|\Psi_{b}\right\rangle$ provide a good description for the Hamiltonian Eq. (3). It is straightforward to get the ground energy of this variational ground state at the point $J=3 J_{1}=3 J_{2}$

$$
\left\langle\Psi_{a}|H| \Psi_{a}\right\rangle=-\frac{3}{2} J N,
$$

where $\mathrm{N}$ is the number of the triangles in the lattice.

It is also interesting to study the properties of the excited states as well as magnetization process of this model. It's well known that for the 2D dimer model, there are magnetization plateaus in the magnetization curve because of the localization of the single triplet excitation [7, 25, 26]. It is natural to ask whether it happens in our model. Notice that for a single trimer, the lowest excitation state is the triplet state, which is the product of a dimer singlet composed of two spins $\left(\frac{1}{\sqrt{3}}(|1,-1\rangle+|-1,1\rangle-|00\rangle)\right.$ and a single spin state $(|1\rangle,|-1\rangle$ or $|0\rangle)$.
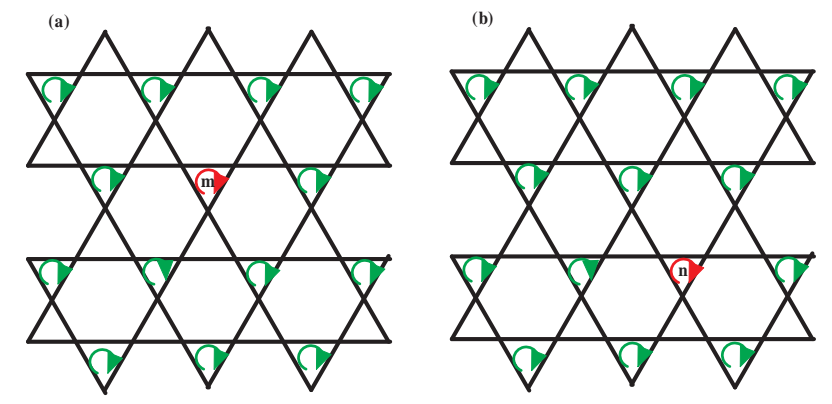

(c)

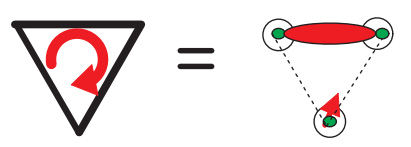

FIG. 3: The triplet excited states: a triplet locates at m (a) and $\mathrm{n}(\mathrm{b}), \mathrm{m}$ and $\mathrm{n}$ are nearest neighbor triplets. A triplet is formed by a dimer of two spin 1 and a single spinon (c)

The triplet state is shown in Fig.3. For convenience, we use $|m\rangle$ denoting the $m$ th trimer singlet being excited to a triplet. To study the low energy excitation of this model. We calculate and find that

$$
\langle m|H| n\rangle=0
$$

for $m \neq n$ and

$$
\langle m|H| m\rangle=-J-\frac{3}{2} J(N-1)
$$

where $H$ is the Hamiltonian (3), and $|m\rangle$ and $|n\rangle$ are shown in Fig.3 (a), (b). Eq.(6) means that the single triplet excitation is almost dispersionless, at least to the order we considered. Eq.(7) means that it is gapped, with a gap of $\frac{1}{2} J$. This dispersionless triplet excitation in our model is different from its one dimensional analogue [27, 28], and it would localize or form a bound pair with other single triplet, just as in the dimer state [29, 30]. Because of the localization of the single triplet excitation, there are magnetization plateaus in the magnetization curve in our model. The naive picture for the magnetization process is shown in Fig.4. Therefore, at least two magnetization plateaus appear at $\mathrm{m} / \mathrm{m}_{\text {sat }}=1 / 2$ and 3/4 corresponding to the phase (b) and (c) in Fig.4. It is known that similar magnetization process and magnetization plateaus have been studied in other Spin-1 systems, such as $\mathrm{S}=1$ spin chain [28], or $\mathrm{S}=1$ Heisenberg model (only including the nearest coupling) in uniform and distorted kagome lattice 31.

However, we have not considered the effect of the interactions between the triplets, which may lead to new plateaus. Further more, it is possible that the higher order interaction would result in the pair of neighboring triplets, similar to the dimer phase [29, 30].

Now we will discuss the stability of our trimer state. Up to now, all of our analysis are based on a special point of our coupling parameters: . At this point, the trimer 

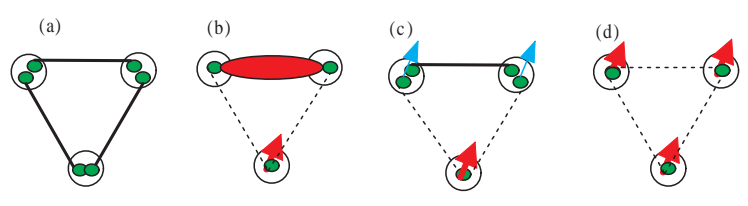

FIG. 4: The magnetization process for a single trimer.(a)The ground state with total spin $S_{\text {total }}=0$.(b)The state with total spin $S_{\text {total }}=1$, it is formed by a dimer and a spinon with $\mathrm{S}=1$ (the bigger red arrowhead). (c) $S_{\text {total }}=2$, the smaller blue arrowhead represents the spinon with $\mathrm{S}=1 / 2$. (d) $S_{\text {total }}=3$, the state with saturated magnetization

state actually provide a good approximation of the exact ground state, and slight perturbation should not change the nature of this ground state because it is a gapped VBS state which can not change by small perturbation unless it can overcome the energy gap, just as in one dimensional AKLT state, introducing small next nearest coupling can't change the nature of 1D VBS state 32] .

Usually there are two kinds of perturbation, the first kind is deviation from the special point of the coupling parameters $J=3 J_{1}=3 J_{2}$, as analyzed above, where slight deviation will not change the nature of the ground state. However, there is another important point: $J_{1}=J_{2}=0$, which corresponds to the $\mathrm{S}=1$ antiferromagnetic Heisenberg model with only nearest coupling. Hida first studied the ground state in this point by means of exact diagonalization and the cluster expansion [17]. It is shown that the ground state in this case is the hexagon singlet solid (HSS) state, rather than the trimer state in our case. The second possible perturbation for this kind of spin-1 system is the spin biquadratic term, which is necessary to construct the 1D AKLT model [9]. However, as shown in Ref. [10], the exact ground state of the AKLT Hamiltonian $\left(3 P^{2}(i, i+1)\right.$ in Eq.(2)) which includes the biquadratic term, actually provides a perfect variational ground state for the pure $\mathrm{S}=1$ Heisenberg Hamiltonian without biquadratic term. Therefore in our case, the perturbation of including the small spin biquadratic term would not change the nature of our trimer state.
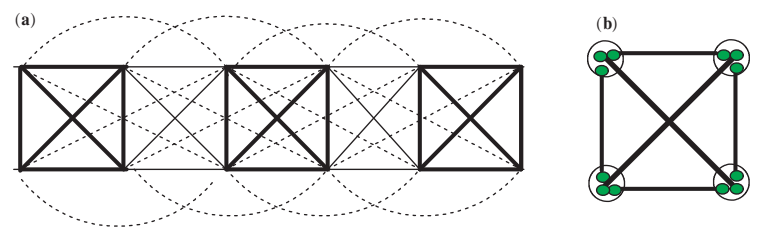

FIG. 5: (a) The cluster state $(\mathrm{N}=4)$ in spin-3/2 ladder. (b)The structure of an $\mathrm{SU}(4)$ cluster singlet. A $3 / 2$ spin can be considered as a symmetrized state of three $1 / 2$ spins. The bold lines represent a singlet for two $1 / 2$ spins

The trimer state is not the only example of our cluster state, another example is the spin- $3 / 2 \mathrm{SU}(4)$ spin ladder [33]. At particular value of the coupling, the ground state of this model is the exact spontaneous plaquette ground states, in which four $3 / 2$ spins form an SU(4) spin singlet plaquette and spontaneously breaking the translational symmetry, as shown in Fig.5. This model is a generalization of the MG model to the spin ladder systems and its ground state belongs to our cluster state with $N=4$.

After completing this work, we learnt that in a recent paper 34], similar topic about the 2D multipartite VBS state has been discussed. Z.C. thanks Flavio S. Nogueira for helpful discussions. This work is partially supported by NSF of China under Grant No. 10574150, MOST grant 2006CB921300 and programs of Chinese Academy of Sciences and Deutscher Akademischer Austausch Dienst (DAAD).
[1] P.W. Anderson, Science. 235, 1196 (1987).

[2] P. Fazekas and P.W. Anderson, Phios. Mag. 30, 432 (1974).

[3] I. Affleck and J.B. Marston, Phys. Rev. B 37, 3774 (1988).

[4] N.Read and S. Sachdev, Phys. Rev. B 42, 4568 (1990); Phys. Rev. Lett. 62, 1694 (1989).

[5] C.K. Majumdar and D.P. Ghosh, J. Math. Phys. 10, 1388 (1969)

[6] B.S. Shastry and B. Sutherland, Physica B 108, 1069 (1981).

[7] H. Kageyama, et al, Phys. Rev. Lett. 82, 3168 (1999).

[8] J.J. Garcia-Ripoll, M.A. Martin-Delgado, and J.I. Cirac, Phys. Rev. Lett. 93, 250405 (2004);

[9] I. Affleck, T. Kennedy, E. H. Lieb, and H. Tasaki, Phys. Rev. Lett. 59, 799 (1987); Commun. Math. Phys. 115, 477 (1988).

[10] D. P. Arovas, A. Auerbach and F. D. M. Haldane, Phys. Rev. Lett. 60, 531 (1988).

[11] Z. Cai, S. Chen, S. P. Kou, Y. Wang, Phys. Rev. B 76,
054443 (2007).

[12] S.K. Yip, Phys. Rev. Lett. 90, 250402 (2003).

[13] S. Chen, Y. Wang, W. Q. Ning, C. Wu and H. Q. Lin, Phys. Rev. B, 74, 174424 (2006).

[14] M.Greiter, S.Rachel, D.Schuricht, Phys. Rev. B, 75, 060401(R) (2007)

[15] M.Greiter, S.Rachel, Phys. Rev. B, 75, 184441 (2007)

[16] J. Solyom and J. Zittartz, Europhys. Lett. 50, 389 (2000).

[17] K. Hida, J. Phys. Soc. Jpn. 69, 4003 (2000); J. Phys. Soc. Jpn. 71, 1027 (2002)

[18] G. Lawes, et al, Phys. Rev. Lett. 93, 247201 (2004).

[19] C. K. Xu and J.E. Moore, Phys. Rev. B 72, 064455 (2005)

[20] M. Levin and X.-G. Wen, Phys. Rev. B 75, 075116 (2007)

[21] C. K. Xu and J. E. Moore, Phys. Rev. B 76, 104427 (2007)

[22] S. V. Isakov and Y.B. Kim, arXiv:cond-mat/0805.2075 (2008).

[23] F. D. M. Haldane, Phys. Rev. Lett. 51, 605 (1983).

[24] H.C. Jiang, F. Kruger, J.E. Moore, D.N. Sheng, J.Zaanen and Z.Y. Weng, arXiv:cond-mat/0901.3141 (2009). 
[25] S. Miyahara and K. Ueda, Phys. Rev. Lett. 82, 3701 (1999).

[26] C. Knetter, A. Bühler, E. Müller-Hartmann and G. S. Uhrig, Phys. Rev. Lett. 85, 3958 (2000).

[27] K. Takano, J. Phys. Soc. Jpn. 63, 4565 (1994).

[28] H. Nakano and M. Takahashi, J. Phys. Soc. Jpn. 67, 1128 (1998).

[29] K. Totsuka, S. Miyahara and K. Ueda, Phys. Rev. Lett. 86, 520 (2001).
[30] T. Momoi, K. Totsuka, Phys. Rev. B 62, 15067 (2000).

[31] K. Hida, J. Phys. Soc. Jpn. 70, 3673 (2001).

[32] H. Nakano and M. Takahashi, Phys. Rev. B 54, 9000 (1994).

[33] S. Chen, C. Wu, S. C. Zhang and Y. Wang, Phys. Rev. B. 72, 214428 (2005).

[34] E. Rico and H.J. Briegel, Ann. Phys. 323, 2115 (2008). 